\title{
Phylogenetic profiling identifies glucosyl-phosphoryl dolichol scramblase candidates
}

\author{
Lucy A. Skrabanek ${ }^{* 1,2}$ and Anant K. Menon ${ }^{\dagger 3}$ \\ ${ }^{1,2}$ Applied Bioinformatics Core, Department of Physiology and Biophysics \\ ${ }^{3}$ Department of Biochemistry, Weill Cornell Medical College, 1300 York Avenue, New York, NY \\ 10065, USA
}

24 October, 2017

\section{Background}

Protein glycosylation is essential for all eukaryotes, from disease-causing protists such as malaria and trypanosomes, to yeast and mammals. Secretory proteins are almost invariably $N$-glycosylated, $O$ - and $C$-mannosylated, and/or GPI-anchored as they enter the lumen of the endoplasmic reticulum (ER).

All ER protein glycosylation reactions occur in the lumen and often involve lumenal mannosylation and glucosylation steps in which mannose and glucose residues are sourced from the glycolipids mannosyl- and glucosyl-phosphoryl dolichol (MPD and GPD, respectively) [1, 2. Paradoxically, these two lipids are synthesized on the cytoplasmic face of the ER and must therefore be flipped across the ER membrane to provide a source of lumenal mannose and glucose. As the spontaneous rate of MPD and GPD flipping is extremely low, specific transporters are needed to facilitate the transbilayer movement of MPD and GPD across the ER membrane at a physiological rate. MPD and GPD transport activities have been demonstrated and characterized in ER microsomes, as well as in vesicles reconstituted with ER membrane proteins [3] 6 . The transport proteins have been shown to be highly structure specific, discriminating between isomers of their lipid substrates, and facilitating lipid movement bidirectionally in an ATP-independent manner (the last point defines them as scramblases, whereas they were previously known as ATP-independent flippases). Although most of the enzymes and co-factors of ER protein glycosylation are known, the molecular identities of the critical dolichol glycolipid scramblases remain a mystery.

Unlike MPD scramblase that is required for all ER protein glycosylation reactions, GPD scramblase is needed exclusively for the synthesis of the glucosylated $N$-glycan precursor $\mathrm{Glc}_{3} \mathrm{Man}_{9} \mathrm{GlcNAc}_{2}$-PP-dolichol (G3M9-DLO). Even though non-glucosylated $N$-glycan precursors are substrates for the protein $N$-glycosylation machinery, the presence of the tri-glucosyl cap - and hence GPD scramblase activity - is critically important for glycosylation efficiency in many eukaryotes, including yeast and humans [7. Two points are noteworthy. (i) Not all organisms have glucose in their $N$-glycan precursor [8]. (ii) While the synthesis of glucosylated $N$-glycan precursors is not essential for the viability of yeast 9, 10, yeast cells that are deficient in G3M9-DLO synthesis display numerous phenotypes including under-glycosylation of proteins, abnormal cell shape and altered susceptibility to a variety of chemicals. In humans, optimization of oligosaccharide transfer efficiency provided by the glucosyl cap is critical as evinced by severe human diseases.

Taking advantage of the fact that not all $N$-glycosylation-competent organisms have glucose in their $N$-glycan precursor, we implemented a bioinformatics approach for assignment of protein function, namely phylogenetic profiling. Using this procedure, we identified a number of polytopic ER membrane proteins as GPD scramblase candidates in yeast.

\footnotetext{
*e-mail: las2017@med.cornell.edu

†e-mail: akm2003@med.cornell.edu
} 


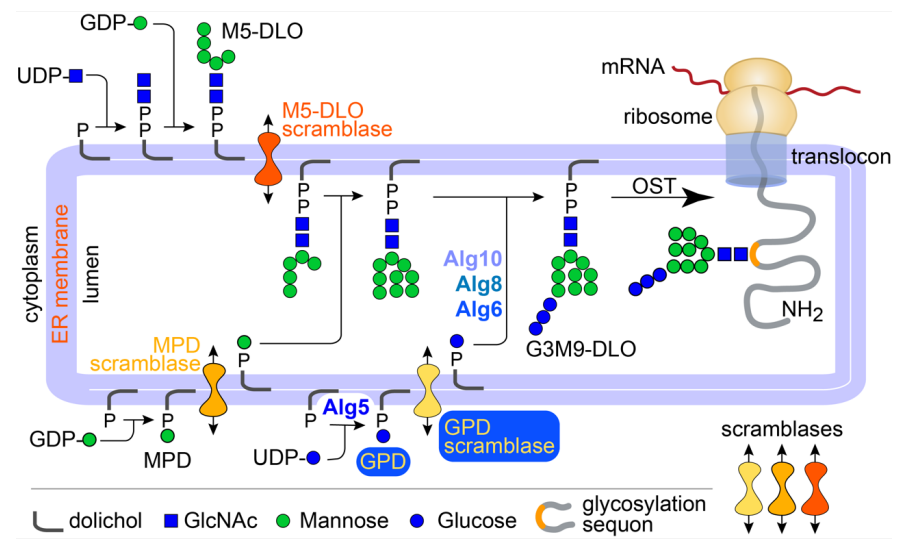

Figure 1: Role of GPD scramblase in G3M9DLO synthesis in yeast. $\mathrm{Glc}_{3} \mathrm{Man}_{9} \mathrm{GlcNAc}_{2}$-PPdolichol (G3M9-DLO; DLO=dolichol-linked oligosaccharide) is synthesized via a series of reactions that begin on the cytoplasmic face of the ER to generate $\mathrm{Man}_{5} \mathrm{GlcNAc}_{2}-$ PP-dolichol (M5-DLO). M5-DLO is translocated across the ER membrane by M5-DLO scramblase. In the lumen, M5-DLO is extended to $\mathrm{Man}_{9} \mathrm{GlcNAc}_{2}-\mathrm{PP}$-dolichol (M9DLO) through the action of MPD-dependent mannosyltransferases and eventually extended to G3M9-DLO. The glucosylation reactions require lumenally oriented GPD. GPD is synthesized from dolichyl-P and UDP-glucose on the cytoplasmic face of the ER by GPD synthase (Alg5), and then moved to the lumen by GPD scramblase. In the lumen, the glucosyltransferases Alg6, Alg8 and Alg10 use GPD to add glucose residues to M9-DLO to generate G3M9-DLO that provides the oligosaccharide used by oligosaccharyltransferase (OST) to $N$-glycosylate proteins. The synthesis, scrambling and consumption of GPD likely form a co-evolving metabolic unit. This premise is the basis of our phylogenetic profiling identification of GPD scramblase candidates.

\section{Results}

\subsection{GPD scramblase candidates identified by phylogenetic profiling}

Phylogenetic profiling predicts protein function based on patterns of protein presence or absence across multiple species (e.g. [11, 12]). If homologs are inherited or lost co-dependently, there is a high chance that they are functionally related or physically interacting, because they are likely to be subject to the same functional constraints, or lack thereof. Proteins with similar phylogenetic profiles tend to be part of a functional unit. A previous study [13] showed that glycosyltransferases are present or absent in organisms in sets. Here we used patterns generated by phylogenetic profiling to identify GPD scramblase candidates. As not all organisms have glucose in their $N$-glycan precursor 8 , we hypothesized that the presence or absence of GPD scramblase in a particular organism will be highly correlated with, but not necessarily identical to, the presence or absence of other proteins of the glucosylation pathway, e.g. the glucosyltransferase Alg6 (Figure 1). We used yeast as a reference organism as it has a well-annotated genome and a complete $N$-glycosylation pathway.

\subsection{Annotated genomes for phylogenetic profiling}

We downloaded 687 annotated genomes from the Ensembl database: 408 fungal, 133 protist, 143 archaeal, 3 higher eukaryotes (dog, mouse, human). We downloaded all the fungal and all the protist genomes from release 27 of the Ensembl database, as well as 143 selected archaeal genomes (those which are also part of the OMA Browser database).

Within this set we identified organisms capable of $N$-glycosylation by using BLAST [14] (E-value threshold 1e-6) to probe for Alg7, the enzyme that initiates DLO synthesis, and Stt3, the catalytic subunit of oligosaccharyltransferase (we identified the Alg7 and Stt3 proteins in Saccharomyces cerevisiae from the SwissProt database as entries P07286.1 and P39007.2, respectively, which correspond to the YBR243C and YGL022W proteins in the Ensembl (SGD) annotation). Only genomes encoding both proteins were retained.

Phylogenetic profiling benefits from an increased amount of input data, but previous studies have shown diminishing returns as greater numbers of genomes are added [15. Thus, we further reduced our dataset by picking a single exemplar for species that were represented by more than one strain. Our final list contained 337 genomes (see Appendix A).

\subsection{Species tree of organisms capable of $N$-glycosylation}

Several authors have suggested that phylogenetic profiles should be ordered by the phylogenetic relatedness of the constituent organisms, e.g., Cokus et al. [16]. The 337 organisms being used in this analysis were imported into 
NCBI's Taxonomy Browser (http://www.ncbi.nlm.nih.gov/Taxonomy/CommonTree/wwwcmt.cgi). Some species were represented in NCBI under a different name, either because the name was spelled slightly differently in NCBI, or because the asexual and sexual stages have different names; these were accordingly changed before import. Table 1 indicates the species which are named differently in the Ensembl and NCBI databases.

\begin{tabular}{|l|l|}
\hline Ensembl nomenclature & NCBI nomenclature \\
\hline Dacryopinax sp & Dacryopinax sp. DJM-731 SS1 \\
Microsporidia sp & Mitosporidium daphniae \\
Paracoccidioides sp & Paracoccidioides sp. 'lutzii' Pb01 \\
Phytomonas sp & Phytomonas sp. EM1 \\
Saccharomycetaceae sp & Saccharomycetaceae sp. 'Ashbya aceri' \\
Pyrenophora triticirepentis & Pyrenophora tritici-repentis \\
Ashbya gossypii & Eremothecium gossypii \\
Canis familiaris & Canis lupus familiaris \\
Ceriporiopsis subvermispora & Gelatoporia subvermispora \\
Dothistroma septosporum & Mycosphaerella pini \\
Magnaporthe poae & Magnaporthiopsis poae \\
Melampsora laricipopulina & Melampsora larici-populina \\
Phaeosphaeria nodorum & Parastagonospora nodorum \\
Pythium vexans & Phytopythium vexans \\
\hline
\end{tabular}

Table 1: Species whose names differ in the Ensembl and NCBI databases.

The phylogenetic representation of the relationship of each of the 337 organisms to each other as determined by the NCBI's Taxonomic Browser was retrieved. The order of the organisms shown in Appendix A reflects the phylogenetic relationship to one another as determined by this method.

\subsection{Profile generation}

We used BLAST [14 to search the protein complement of each of the 337 genomes for homologs to all 6,692 yeast proteins. This was done three separate times, each time using a different E-value threshold cutoff (no threshold, 1e-2, and 1e-10 for our most stringent match). Three types of profiles were generated: (i) BRH (best reciprocal hit), a binary profile, where the presence or absence of each yeast protein is predicted for each organism. For a yeast protein to be marked as existing in organism $\mathrm{O}$, the protein in organism $\mathrm{O}$ that is the highest scoring hit to the yeast protein must also find that same yeast protein as one of its top two hits when searched against the yeast proteome. (ii) Score profile, a quantitative homology measure, where the score of the top hit to the yeast protein is divided by the score of its self-match (i.e., the score when the yeast protein is aligned to itself). This normalized score is a continuous variable from 0 to $1(0=$ non-existence; 1 = perfect match). (iii) $\mathbf{E}$-value profile, calculated by taking the $1 / \log 10$ (E-value score), and capping the maximum value at 1 . The E-value profile is on a $0-1$ scale, where 0 is a perfect match, and 1 is non-existence. The profile of Alg5 (GPD synthase) [9, 10] was more promiscuous than expected (see below), so as bait we instead used the GPD-dependent glucosyltransferase Alg6 [9]. Distances of all profiles were calculated relative to Alg6, using a Jaccard-like metric for the BRH profile, and the Canberra distance metric for the others.

\section{$2.5 \quad$ Filters}

Annotations for each yeast protein were obtained from the Saccharomyces Genome Database (SGD) (http:// downloads.yeastgenome.org/curation/chromosomal_feature/SGD_features.tab). Only records pertaining to ORFs were retained. Transmembrane domains were predicted for all yeast proteins using the TMHMM server (http://www.cbs.dtu.dk/services/TMHMM/ [17]). The results were filtered to include only membrane proteins, and further refined to prioritize only multispanning proteins ( $\geq 3$ transmembrane domains). We also filtered out proteins that were not found in the three higher eukaryotes. A final manual filter restricted the list to proteins that are known to be ER-localized, and those whose localization is ambiguous.

For each BLAST E-value threshold, we listed the top 50 hits that were identified in all three profile-generating methods (Appendix B) and ranked them according to the lowest rank that a protein has in any of the three lists. Although the lists differ slightly, the top candidates are common to all: (in rank order) Alg8, Mns1, Ale1, Erg24, Erc1, Ybr220C, Erg3, Gwt1, Alg2, Scs7, Ste24, Erg11, Sur2, Ydr338C, Alg3, Alg9, and Ykr051W. Depending on the 
outcome of our biochemical analyses, we may pick additional candidates from the ranked lists that we generated, potentially including proteins that span the membrane only once. Such proteins may oligomerize to generate a pseudo-polytopic membrane protein that could have a transport function.

The presence of Alg8 as our top hit is unsurprising because it is required for synthesis of the glucose cap in G3M9-DLO and would be expected to co-evolve with Alg6. Three candidates (Ybr220C, Ydr338C, Ykr051W) are annotated as proteins of unknown function, two (Erc1, Ydr338C) are homologous to proteins that belong to the MOP exporter superfamily, and all except three (Gwt1, Alg2, Erg11) are non-essential for growth, consistent with glucosylation being non-essential in yeast. Interestingly, most have a lipid-related function, e.g. Ale1 is a lysophospholipid acyltransferase and Scs7 is a sphingolipid fatty acid $\alpha$-hydroxylase, and three (Erg24, Ste24, Erg11) were identified in photocrosslinking studies using dolichyl phosphate analogs [18].

\subsection{Notes on the profiling method and results}

Phylogenetic profiling is a powerful method to identify functionally associated, co-evolving proteins such as enzymes that catalyze sequential steps in a biochemical pathway. While the method identifies the most significant co-evolutionary events, the generated profiles may not always match exactly. The most common causes for imperfect matches are a) incomplete protein annotation of a genome, b) different rates of evolution in independent lineages which may cause evolutionarily more distant proteins to outscore (in BLAST) the proteins which are actually closer, and (c) the possibility that in some organisms, a protein may have another, unrelated, function [11, 19].

Thus, Alg5 is found in a few organisms that do not synthesize glucosylated DLOs, suggesting novel roles for GPD [20] and/or an alternate function for Alg5 in these organisms. Likewise, the glucosyltransferase Alg8 is present in every Alg6-positive genome (268 of the 337 genomes we analyzed are Alg6-positive) as expected, but it is also present in 3 genomes (e.g. Toxoplasma gondii) that do not have Alg6. As Alg8 cannot add glucose unless Alg6 has first acted, and it has no other known function, its presence in these three genomes may be an evolutionary remnant. Another reason that profiles may not match exactly is if the GPD scramblase protein has another function, and is therefore subject to selective pressure unrelated to the glucosylation pathway. In this case, the candidate protein should be present if Alg6 is present, but may also be present when Alg6 is absent. For example, Erg24 is found in 19 of the 69 genomes that do not have Alg6. These genomes include those of trypanosomatids (e.g. Trypanosoma brucei) that do not synthesize glucosylated DLOs, but require the C14 sterol reductase activity of Erg24 to synthesize sterols. Thus, Erg24 may be a bifunctional protein with both GPD scramblase and sterol reductase activities. Despite the not-always-perfect profile matches, it is important to recognize that the power of the phylogenetic profiling approach used here lies in its ability to generate an unbiased, ranked list of candidates that allow us to prioritize our efforts to evaluate potential scramblase functions of the candidates.

\section{Summary}

Our top GPD scramblase candidates (in rank order, those in bold are essential for yeast growth) identified by phylogenetic profiling are Alg8, Mns1, Ale1, Erg24, Erc1, Ybr220C, Erg3, Gwt1, Alg2, Scs7, Ste24, Erg11, Sur2, Ydr338C, Alg3, Alg9, and Ykr051W. We are currently evaluating these candidates via in vitro and in vivo tests to identify the scramblase. This preprint will be updated once experimental data are available.

\section{Acknowledgements}

We thank John Samuelson (Boston University Medical School) for his initial efforts on this project, and Sam Canis for stimulation. This work was supported by NIH grant NS093457 and a grant (P 61397-009) from the Alice Bohmfalk Charitable Trust (both to AKM).

\section{References}

[1] B Schenk, F Fernandez, and CJ Waechter. The ins(ide) and out(side) of dolichyl phosphate biosynthesis and recycling in the endoplasmic reticulum. Glycobiology, 11(5):61R-70R, 2001.

[2] S Sanyal and AK Menon. Flipping lipids: why an' what's the reason for? ACS Chem Biol, 4(11):895-909, 2009.

[3] JS Rush and CJ Waechter. Transmembrane movement of a water-soluble analogue of mannosylphosphoryldolichol is mediated by an endoplasmic reticulum protein. J Cell Biol, 130(3):529-536, 1995. 
[4] JS Rush and CJ Waechter. Functional reconstitution into proteoliposomes and partial purification of a rat liver er transport system for a water-soluble analogue of mannosylphosphoryldolichol. Biochemistry, 43(23): $7643-7652,2004$.

[5] JS Rush, K van Leyen, O Ouerfelli, B Wolucka, and CJ Waechter. Transbilayer movement of glc-p-dolichol and its function as a glucosyl donor: protein-mediated transport of a water-soluble analog into sealed er vesicles from pig brain. Glycobiology, 8(12):1195-1205, 1998.

[6] S Sanyal and AK Menon. Stereoselective transbilayer translocation of mannosyl phosphoryl dolichol by an endoplasmic reticulum flippase. Proc Natl Acad Sci U S A, 107(25):11289-11294, 2010.

[7] SJ Turco, B Stetson, and PW Robbins. Comparative rates of transfer of lipid-linked oligosaccharides to endogenous glycoprotein acceptors in vitro. Proc Natl Acad Sci U S A, 74(10):4411-4414, 1977.

[8] J Samuelson and PW Robbins. Effects of n-glycan precursor length diversity on quality control of protein folding and on protein glycosylation. Semin Cell Dev Biol, 41:121-128, 2015.

[9] KW Runge, TC Huffaker, and PW Robbins. Two yeast mutations in glucosylation steps of the asparagine glycosylation pathway. J Biol Chem, 259(1):412-417, 1984.

[10] S Heesen, L Lehle, A Weissmann, and M Aebi. Isolation of the alg5 locus encoding the udp-glucose:dolichylphosphate glucosyltransferase from saccharomyces cerevisiae. Eur J Biochem, 224(1):71-79, 1994.

[11] M Pellegrini, EM Marcotte, MJ Thompson, D Eisenberg, and TO Yeates. Assigning protein functions by comparative genome analysis: protein phylogenetic profiles. Proc Natl Acad Sci U S A, 96(8):4285-4288, 1999.

[12] G Dey, A Jaimovich, SR Collins, A Seki, and T Meyer. Systematic discovery of human gene function and principles of modular organization through phylogenetic profiling. Cell Reports, 15:S2211-1247, 2015.

[13] J Samuelson, S Banerjee, P Magnelli, J Cui, DJ Kelleher, R Gilmore, and PW Robbins. The diversity of dolichol-linked precursors to asn-linked glycans likely results from secondary loss of sets of glycosyltransferases. Proc Natl Acad Sci U S A, 102(5):1548-1553, 2005.

[14] SF Altschul, TL Madden, AA Schäffer, J Zhang, Z Zhang, W Miller, and DJ Lipman. Gapped blast and psi-blast: a new generation of protein database search programs. Nucleic Acids Res, 25(17):3389-3402, 1997.

[15] N Škunca and C Dessimoz. Phylogenetic profiling: How much input data is enough? PLoS One, 10(2):e0114701, 2015.

[16] S Cokus, S Mizutani, and M Pellegrini. An improved method for identifying functionally linked proteins using phylogenetic profiles. BMC Bioinformatics, 8(Suppl 4):S7, 2007.

[17] A Krogh, B Larsson, G von Heijne, and ELL Sonnhammer. Predicting transmembrane protein topology with a hidden markov model: application to complete genomes. J Mol Biol, 305(3):567-580, 2001.

[18] JS Rush, T Subramanian, KL Subramanian, FO Onono, CJ Waechter, and HP Spielmann. Novel citronellylbased photoprobes designed to identify er proteins interacting with dolichyl phosphate in yeast and mammalian cells. Curr Chem Biol, 9(2):123-141, 2015.

[19] MK Basu, JD Selengut, and DH Haft. Prophylo: partial phylogenetic profiling to guide protein family construction and assignment of biological process. BMC Bioinformatics, 12:434-447, 2011.

[20] KA Grabinska, SK Ghosh, Z Guan, J Cui, CR Raetz, PW Robbins, and J Samuelson. Dolichyl-phosphateglucose is used to make o-glycans on glycoproteins of trichomonas vaginalis. Eukaryotic Cell, 7(8):1344-1351, 2008. 


\section{Appendix A: List of organisms used in phylogenetic profiling analysis, ordered by phylogenetic relatedness}

1. Guillardia theta

2. Bigelowiella natans

3. Reticulomyxa filosa

4. Perkinsus marinus atcc 50983

5. Oxytricha trifallax gca 000295675

6. Stylonychia lemnae

7. Tetrahymena thermophila

8. Paramecium tetraurelia

9. Babesia equi strain wa

10. Babesia bovis

11. Plasmodium reichenowi

12. Plasmodium falciparum

13. Plasmodium inui san antonio 1

14. Plasmodium vivax

15. Plasmodium knowlesi

16. Plasmodium cynomolgi strain $b$

17. Plasmodium yoelii yoelii

18. Plasmodium vinckei petteri

19. Plasmodium chabaudi

20. Plasmodium berghei

21. Gregarina niphandrodes

22. Hammondia hammondi

23. Toxoplasma gondii

24. Cryptosporidium muris rn66

25. Cryptosporidium parvum iowa ii

26. Eimeria necatrix

27. Eimeria tenella gca 000499545

28. Naegleria gruberi

29. Polysphondylium pallidum pn500

30. Dictyostelium fasciculatum

31. Dictyostelium purpureum

32. Entamoeba nuttalli p19

33. Entamoeba dispar saw760

34. Entamoeba invadens ip1

35. Entamoeba histolytica

36. Acanthamoeba castellanii str neff

37. Spironucleus salmonicida

38. Giardia intestinalis

39. Giardia lamblia

40. Trichomonas vaginalis g3

41. Phytomonas sp isolate em1

42. Angomonas deanei

43. Trypanosoma rangeli sc58

44. Trypanosoma cruzi

45. Trypanosoma brucei

46. Leishmania mexicana mhom gt 2001 u1103

47. Leishmania major

48. Leishmania infantum jpcm5

49. Leishmania donovani

50. Leishmania panamensis

51. Leishmania braziliensis mhom br 75 m2904

52. Salpingoeca rosetta

53. Monosiga brevicollis $\mathrm{mx} 1$

54. Canis familiaris

55. Mus musculus

56. Homo sapiens

57. Rhizophagus irregularis daom 181602

58. Microsporidia sp ugp3
59. Nematocida parisii ertm1

60. Rozella allomycis csf55

61. Batrachochytrium dendrobatidis jam81

62. Wallemia ichthyophaga exf 994

63. Wallemia sebi cbs 63366

64. Mixia osmundae iam 14324

65. Melampsora laricipopulina

66. Puccinia triticina

67. Puccinia graminis

68. Rhodosporidium toruloides np11

69. Microbotryum violaceum

70. Tilletiaria anomala ubc 951

71. Pseudozyma brasiliensis

72. Pseudozyma hubeiensis sy 62

73. Pseudozyma aphidis dsm 70725

74. Pseudozyma antarctica

75. Sporisorium reilianum

76. Ustilago hordei

77. Ustilago maydis

78. Dacryopinax sp djm 731 ss1

79. Heterobasidion irregulare tc 321

80. Rhizoctonia solani $123 \mathrm{e}$

81. Botryobasidium botryosum fd 172 ss1

82. Tulasnella calospora mut 4182

83. Gloeophyllum trabeum atcc 11539

84. Serendipita vermifera maff 305830

85. Piriformospora indica dsm 11827

86. Fibroporia radiculosa

87. Phanerochaete carnosa hhb $10118 \mathrm{sp}$

88. Phlebiopsis gigantea $110611 \mathrm{cr} 56$

89. Ceriporiopsis subvermispora b

90. Fomitopsis pinicola fp 58527 ss1

91. Trametes cinnabarina

92. Jaapia argillacea mucl 33604

93. Piloderma croceum f 1598

94. Paxillus rubicundulus ve08 $2 \mathrm{~h} 10$

95. Serpula lacrymans var lacrymans s7 3

96. Scleroderma citrinum foug a

97. Pisolithus microcarpus 441

98. Pisolithus tinctorius marx 270

99. Suillus luteus uh slu $\operatorname{lm} 8 \mathrm{n} 1$

100. Moniliophthora roreri mca 2997

101. Galerina marginata cbs 33988

102. Hebeloma cylindrosporum $\mathrm{h} 7$

103. Amanita muscaria koide bx008

104. Laccaria amethystina laam 081

105. Laccaria bicolor $s 238 \mathrm{n} h 82$

106. Coprinopsis cinerea okayama7 130

107. Agaricus bisporus var bisporus h97

108. Schizophyllum commune h4 8

109. Pleurotus ostreatus pc15

110. Trichosporon asahii var asahii cbs 2479

111. Cryptococcus gattii r265

112. Cryptococcus neoformans

113. Tuber melanosporum

114. Dactylellina haptotyla cbs 20050

115. Arthrobotrys oligospora atcc 24927

116. Pseudogymnoascus destructans 2063121 
117. Pseudogymnoascus pannorum vkm f 103

118. Oidiodendron maius zn

119. Marssonina brunnea f sp multigermtubi mb $\mathrm{m} 1$

120. Glarea lozoyensis atcc 20868

121. Sclerotinia borealis $\mathrm{f} 4157$

122. Botrytis cinerea

123. Erysiphe necator

124. Blumeria graminis

125. Pestalotiopsis fici w106 1

126. Eutypa lata ucrel1

127. Togninia minima ucrpa7

128. Grosmannia clavigera kw1407

129. Ophiostoma piceae uamh 11346

130. Sporothrix brasiliensis 5110

131. Sporothrix schenckii atcc 58251

132. Magnaporthe oryzae

133. Magnaporthe poae

134. Gaeumannomyces graminis

135. Myceliophthora thermophila atcc 42464

136. Chaetomium thermophilum var thermophilum dsm 1495

137. Chaetomium globosum cbs 14851

138. Thielavia terrestris nrrl 8126

139. Podospora anserina s mat

140. Sordaria macrospora

141. Neurospora tetrasperma fgsc 2508

142. Neurospora crassa

143. Scedosporium apiospermum

144. Verticillium alfalfae vams 102

145. Verticillium dahliae

146. Colletotrichum sublineola

147. Colletotrichum fioriniae pj7

148. Colletotrichum gloeosporioides

149. Colletotrichum higginsianum

150. Colletotrichum graminicola

151. Colletotrichum orbiculare

152. Stachybotrys chlorohalonata ibt 40285

153. Stachybotrys chartarum ibt 40288

154. Beauveria bassiana arsef 2860

155. Cordyceps militaris $\mathrm{cm} 01$

156. Trichoderma atroviride imi 206040

157. Trichoderma reesei

158. Trichoderma virens

159. Fusarium solani

160. Fusarium pseudograminearum

161. Fusarium graminearum

162. Fusarium oxysporum

163. Fusarium verticillioides

164. Fusarium fujikuroi

165. Torrubiella hemipterigena

166. Metarhizium majus arsef 297

167. Metarhizium robertsii

168. Metarhizium brunneum arsef 3297

169. Metarhizium guizhouense arsef 977

170. Metarhizium acridum cqma 102

171. Metarhizium album arsef 1941

172. Metarhizium anisopliae

173. Claviceps purpurea 201

174. Ustilaginoidea virens

175. Acremonium chrysogenum atcc 11550

176. Endocarpon pusillum z07020

177. Cyphellophora europaea cbs 101466
178. Rhinocladiella mackenziei cbs 65093

179. Cladophialophora psammophila cbs 110553

180. Cladophialophora immunda

181. Cladophialophora yegresii cbs 114405

182. Cladophialophora bantiana cbs 17352

183. Cladophialophora carrionii cbs 16054

184. Coniosporium apollinis cbs 100218

185. Fonsecaea pedrosoi cbs 27137

186. Exophiala aquamarina cbs 119918

187. Exophiala sideris

188. Exophiala xenobiotica

189. Exophiala oligosperma

190. Exophiala mesophila

191. Exophiala spinifera

192. Exophiala dermatitidis nih ut8656

193. Capronia coronata cbs 61796

194. Capronia epimyces cbs 60696

195. Capronia semiimmersa

196. Byssochlamys spectabilis no 5

197. Talaromyces marneffei atcc 18224

198. Talaromyces stipitatus atcc 10500

199. Neosartorya fischeri

200. Penicillium rubens wisconsin 541255

201. Penicillium oxalicum 1142

202. Penicillium solitum

203. Penicillium italicum

204. Penicillium digitatum pd1

205. Penicillium expansum

206. Aspergillus fumigatus

207. Aspergillus ruber cbs 135680

208. Aspergillus nidulans

209. Aspergillus terreus

210. Aspergillus oryzae

211. Aspergillus niger

212. Aspergillus flavus

213. Aspergillus clavatus

214. Coccidioides posadasii str silveira

215. Paracoccidioides sp lutzii pb01

216. Paracoccidioides brasiliensis pb03

217. Uncinocarpus reesii 1704

218. Microsporum gypseum cbs 118893

219. Arthroderma otae cbs 113480

220. Arthroderma benhamiae cbs 112371

221. Trichophyton soudanense cbs 45261

222. Trichophyton equinum cbs 12797

223. Trichophyton verrucosum hki 0517

224. Trichophyton interdigitale $\mathrm{h} 6$

225. Trichophyton tonsurans cbs 112818

226. Trichophyton rubrum d6

227. Blastomyces dermatitidis atcc 18188

228. Histoplasma capsulatum h88

229. Verruconis gallopava

230. Neofusicoccum parvum ucrnp2

231. Macrophomina phaseolina ms6

232. Baudoinia compniacensis uamh 10762

233. Zymoseptoria tritici

234. Sphaerulina musiva so2202

235. Pseudocercospora fijiensis cirad 86

236. Dothistroma septosporum

237. Aureobasidium subglaciale exf 2481

238. Aureobasidium melanogenum cbs 110374

239. Aureobasidium pullulans exf 150 
240. Phaeosphaeria nodorum

241. Leptosphaeria maculans

242. Setosphaeria turcica et 28 a

243. Pyrenophora teres

244. Pyrenophora triticirepentis

245. Bipolaris oryzae atcc 44560

246. Bipolaris sorokiniana nd90pr

247. Bipolaris victoriae fi3

248. Bipolaris zeicola 26 r 13

249. Bipolaris maydis atcc 48331

250. Kuraishia capsulata cbs 1993

251. Clavispora lusitaniae atcc 42720

252. Yarrowia lipolytica

253. Saccharomyces arboricola h 6

254. Saccharomycetaceae sp ashbya aceri

255. Kazachstania naganishii cbs 8797

256. Kazachstania africana cbs 2517

257. Lachancea lanzarotensis

258. Lachancea thermotolerans cbs 6340

259. Tetrapisispora blattae cbs 6284

260. Tetrapisispora phaffii cbs 4417

261. Vanderwaltozyma polyspora dsm 70294

262. Eremothecium cymbalariae dbvpg 7215

263. Ashbya gossypii

264. Kluyveromyces lactis

265. Naumovozyma dairenensis cbs 421

266. Naumovozyma castellii cbs 4309

267. Candida glabrata

268. Zygosaccharomyces rouxii

269. Zygosaccharomyces bailii isa 1307

270. Torulaspora delbrueckii

271. Wickerhamomyces ciferrii

272. Komagataella pastoris

273. Spathaspora passalidarum nrrl y 27907

274. Candida tenuis atcc 10573

275. Lodderomyces elongisporus nrrl yb 4239

276. Candida orthopsilosis co 90125

277. Candida dubliniensis cd36

278. Candida tropicalis mya 3404

279. Candida albicans wo 1

280. Debaryomyces hansenii cbs767

281. Meyerozyma guilliermondii atcc 6260

282. Scheffersomyces stipitis cbs 6054

283. Millerozyma farinosa cbs 7064

284. Ogataea parapolymorpha dl 1

285. Pichia kudriavzevii

286. Pneumocystis murina b123

287. Schizosaccharomyces cryophilus

288. Schizosaccharomyces octosporus
289. Schizosaccharomyces japonicus

290. Schizosaccharomyces pombe

291. Aureococcus anophagefferens

292. Blastocystis hominis

293. Albugo laibachii

294. Aphanomyces invadans

295. Aphanomyces astaci

296. Saprolegnia diclina vs20

297. Saprolegnia parasitica cbs 22365

298. Pythium iwayamai

299. Pythium arrhenomanes

300. Pythium ultimum

301. Pythium aphanidermatum

302. Pythium irregulare

303. Pythium vexans

304. Hyaloperonospora arabidopsidis

305. Phytophthora kernoviae

306. Phytophthora ramorum

307. Phytophthora lateralis

308. Phytophthora sojae

309. Phytophthora parasitica

310. Phytophthora infestans

311. Thalassiosira oceanica

312. Thalassiosira pseudonana

313. Phaeodactylum tricornutum

314. Galdieria sulphuraria

315. Chondrus crispus

316. Nitrosopumilus maritimus scm1

317. Caldisphaera lagunensis dsm 15908

318. Metallosphaera cuprina ar 4

319. Metallosphaera sedula

320. Sulfolobus tokodaii str 7

321. Sulfolobus islandicus hve10 4

322. Sulfolobus solfataricus 982

323. Sulfolobus acidocaldarius dsm 639

324. Hyperthermus butylicus dsm 5456

325. Thermogladius cellulolyticus 1633

326. Ignicoccus hospitalis kin4 i

327. Staphylothermus hellenicus dsm 12710

328. Staphylothermus marinus f1

329. Desulfurococcus kamchatkensis $1221 \mathrm{n}$

330. Desulfurococcus mucosus dsm 2162

331. Thermofilum pendens hrk 5

332. Aciduliprofundum boonei t469 gca 0000256651

333. Methanosaeta thermophila pt

334. Methanothermobacter marburgensis str marburg

335. Methanobrevibacter ruminantium $\mathrm{m} 1$

336. Methanosphaera stadtmanae dsm 3091

337. Methanothermus fervidus dsm 2088

\section{Appendix B: Ranked top 50 genes for each BLAST E-value threshold}

\section{Ranked list, Top50 (TM-containing, ER-localized only), BLAST threshold: 0}

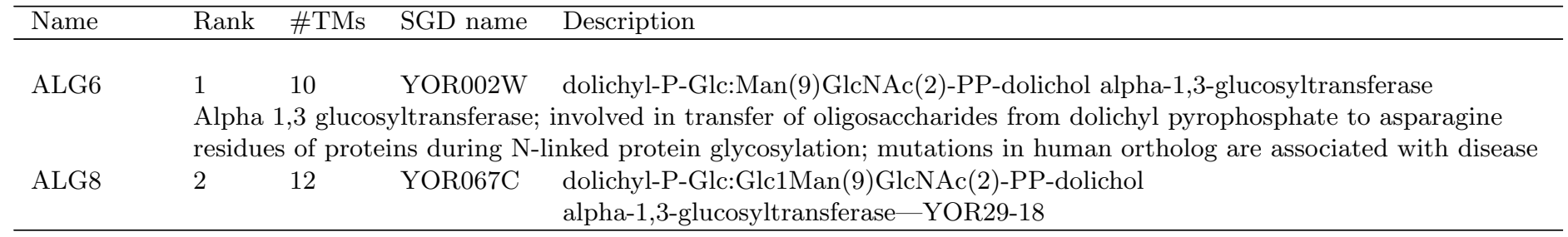




\begin{tabular}{llll}
\hline Name & Rank \#TMs & SGD name Description \\
\hline
\end{tabular}

MNS1 $4 \quad 1 \quad$ YJR131W mannosyl-oligosaccharide 1,2-alpha-mannosidase

Glucosyl transferase; involved in N-linked glycosylation; adds glucose to the dolichol-linked oligosaccharide precursor prior to transfer to protein during lipid-linked oligosaccharide biosynthesis; similar to Alg6p Alpha-1,2-mannosidase; involved in ER-associated protein degradation (ERAD); catalyzes the removal of one mannose residue from a glycosylated protein, converting the modification from Man9GlcNAc to Man8GlcNAc; catalyzes the last step in glycoprotein maturation in the ER and is critical for ER protein degradation

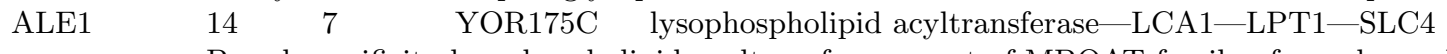
Broad-specificity lysophospholipid acyltransferase; part of MBOAT family of membrane-bound O-acyltransferases; key component of Lands cycle; may have role in fatty acid exchange at sn-2 position of mature glycerophospholipids ERG24 $18 \quad 8 \quad$ YNL280C delta(14)-sterol reductase

C-14 sterol reductase; acts in ergosterol biosynthesis; mutants accumulate the abnormal sterol ignosterol (ergosta-8,14 dienol), and are viable under anaerobic growth conditions but inviable on rich medium under aerobic conditions

AVT3 $35 \quad 10 \quad$ YKL146W YKL146W

Vacuolar transporter; exports large neutral amino acids from the vacuole; member of a family of seven S. cerevisiae genes (AVT1-7) related to vesicular GABA-glycine transporters

$\begin{array}{lllll}\text { CSC1 } & 39 & 11 & \text { YLR241W } & \text { YLR241W }\end{array}$ Calcium permeable gated cation channel; may be involved in detoxification; similar to Arabidopsis CSC1

$\begin{array}{llll}\text { BOR1 } & 40 & 10 & \text { YNL275W } \\ \text { YNL275W }\end{array}$

Boron efflux transporter of the plasma membrane; binds HCO3-, I-, Br-, NO3- and Cl-; has similarity to the characterized boron efflux transporter A. thaliana BOR1

$\begin{array}{lllll}\text { ERG11 } & 45 & 2 & \text { YHR007C sterol 14-demethylase-CYP51 }\end{array}$

Lanosterol 14-alpha-demethylase; catalyzes C-14 demethylation of lanosterol to form 4,4"-dimethyl

cholesta-8,14,24-triene-3-beta-ol in ergosterol biosynthesis pathway; transcriptionally down-regulated when ergosterol is in excess; member of cytochrome P450 family; associated and coordinately regulated with the P450 reductase Ncp1p

$\begin{array}{lllll}\text { ERV46 } & 46 & 1 & \text { YAL042W } & \text { FUN9 }\end{array}$

Protein localized to COPII-coated vesicles; forms a complex with Erv41p; involved in the membrane fusion stage of transport

ERG3 $\quad 50 \quad 3 \quad$ YLR056W C-5 sterol desaturase-PSO6-SYR1

C-5 sterol desaturase; glycoprotein that catalyzes the introduction of a C-5(6) double bond into episterol, a precursor in ergosterol biosynthesis; transcriptionally down-regulated when ergosterol is in excess; mutants are viable, but cannot grow on non-fermentable carbon sources; substrate of HRD ubiquitin ligase

$\begin{array}{lllll}\text { YPR003C } & 52 & 10 & \text { YPR003C } \quad \text { YPR003C }\end{array}$ Putative sulfate permease; physically interacts with Hsp82p; green fluorescent protein (GFP)-fusion protein localizes to the ER; YPR003C is not an essential gene

STE24 $64 \quad 4 \quad$ YJR117W zinc metalloprotease-PIO2-AFC1

Highly conserved zinc metalloprotease; functions in two steps of a-factor maturation, C-terminal CAAX proteolysis and the first step of N-terminal proteolytic processing; contains multiple transmembrane spans; human homolog ZMPSTE24 implicated in mandibuloacral dysplasia (MAD), and can complement yeast null mutant

PMT5 $65 \quad 11 \quad$ YDL093W putative dolichyl-phosphate-mannose-protein mannosyltransferase PMT5 Protein O-mannosyltransferase; transfers mannose residues from dolichyl phosphate-D-mannose to protein serine/threonine residues; acts in a complex with Pmt3p, can instead interact with Pmt2p in some conditions; target for new antifungals

ALG1 $\quad 67 \quad 1 \quad$ YBR110W chitobiosyldiphosphodolichol beta-1,4 mannosyltransferase Mannosyltransferase; involved in asparagine-linked glycosylation in the endoplasmic reticulum (ER); essential for viability; human homolog ALG1 complements yeast null mutant

$\begin{array}{llll}\text { AUR1 } & 67 & 6 & \text { YKL004W inositol phosphorylceramide synthase }\end{array}$

Phosphatidylinositol:ceramide phosphoinositol transferase; required for sphingolipid synthesis; can mutate to confer aureobasidin A resistance; also known as IPC synthase

$\begin{array}{lllll}\text { ZRT2 } & 72 & 7 & \text { YLR130C low-affinity } \mathrm{Zn}(2+) \text { transporter ZRT2 }\end{array}$

Low-affinity zinc transporter of the plasma membrane; transcription is induced under low-zinc conditions by the Zap1p transcription factor

$\begin{array}{llll}\text { ZRT1 } & 80 & 8 & \text { YGL255W high-affinity } \mathrm{Zn}(2+) \text { transporter ZRT1 }\end{array}$

High-affinity zinc transporter of the plasma membrane; responsible for the majority of zinc uptake; transcription is induced under low-zinc conditions by the Zap1p transcription factor

$\begin{array}{lllll}\text { ATG15 } & 83 & 1 & \text { YCR068W triglyceride lipase ATG15-CVT17-AUT5 }\end{array}$

Putative lipase required for lysis of autophagic and Cvt bodies; targeted to intravacuolar vesicles during autophagy via the multivesicular body (MVB) pathway; required for the maintenance of lipid droplet quantity after the diauxic shift; regulates lipolysis 


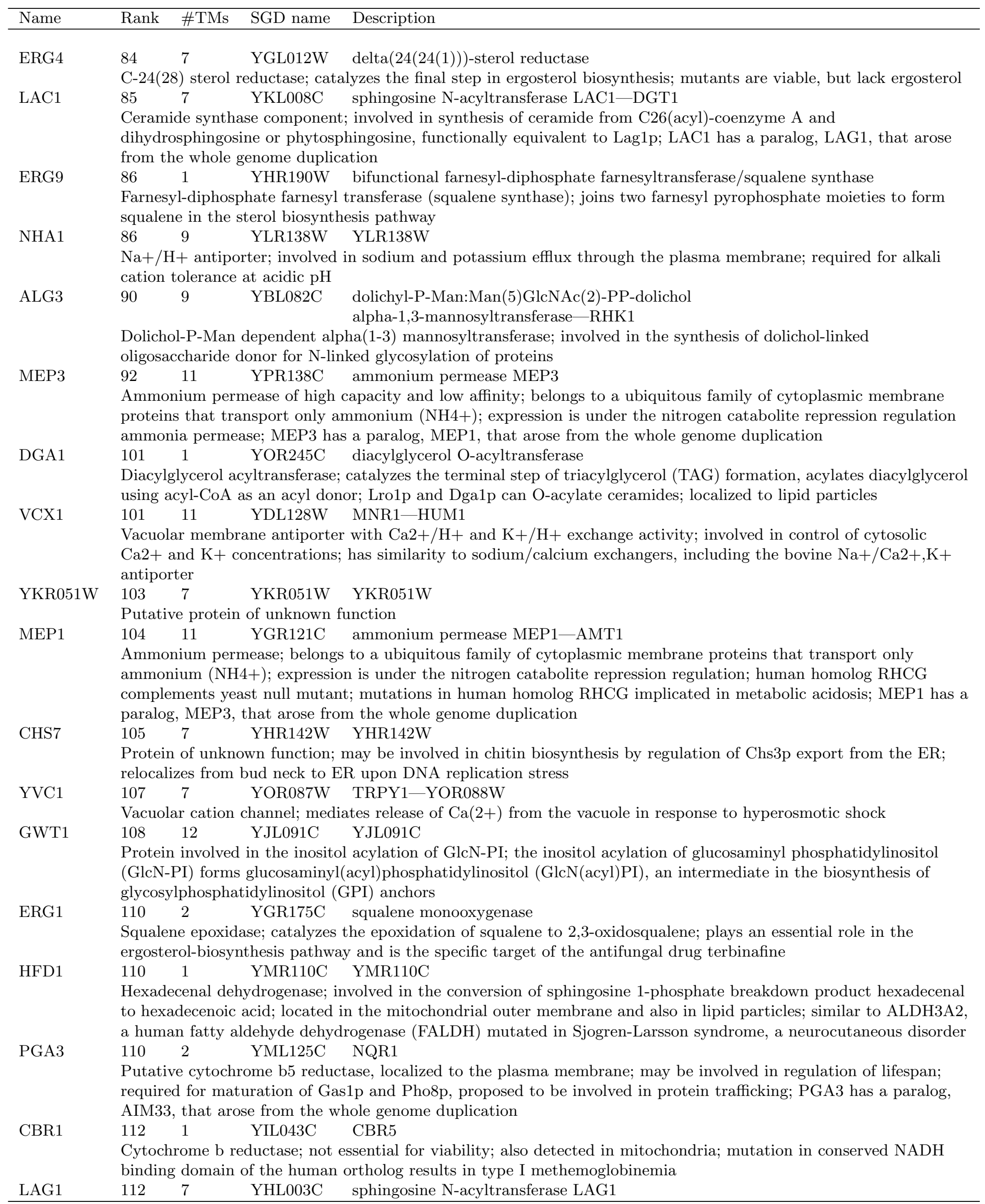




\begin{tabular}{|c|c|}
\hline Name & \#TMs $\quad$ SGD name \\
\hline & $\begin{array}{l}\text { Ceramide synthase component; involved in synthesis of ceramide from C26(acyl)-coenzyme A and } \\
\text { dihydrosphingosine or phytosphingosine, functionally equivalent to Lac1p; forms ER foci upon DNA replication } \\
\text { stress; homolog of human CERS2, a tumor metastasis suppressor gene whose silencing enahnces invasion/metastasis } \\
\text { of prostate cancer cells; LAG1 has a paralog, LAC1, that arose from the whole genome duplication }\end{array}$ \\
\hline \multirow[t]{2}{*}{ ERV29 } & $114 \quad 7 \quad$ YGR284C YGR284C \\
\hline & $\begin{array}{l}\text { Protein localized to COPII-coated vesicles; involved in vesicle formation and incorporation of specific secretory } \\
\text { cargo; protein abundance increases in response to DNA replication stress }\end{array}$ \\
\hline \multirow[t]{2}{*}{ HNM1 } & $114 \quad 12 \quad$ YGL077C $\quad$ CTR1 \\
\hline & $\begin{array}{l}\text { Plasma membrane transporter for choline, ethanolamine, and carnitine; involved in the uptake of nitrogen mustard } \\
\text { and the uptake of glycine betaine during hypersaline stress; co-regulated with phospholipid biosynthetic genes and } \\
\text { negatively regulated by choline and myo-inositol }\end{array}$ \\
\hline \multirow[t]{2}{*}{ OLE1 } & $114 \quad 4 \quad$ YGL055W stearoyl-CoA 9-desaturase-MDM2 \\
\hline & $\begin{array}{l}\text { Delta(9) fatty acid desaturase; required for monounsaturated fatty acid synthesis and for normal distribution of } \\
\text { mitochondria }\end{array}$ \\
\hline \multirow[t]{2}{*}{ FTH1 } & $115 \quad 7 \quad$ YBR207W $\quad$ YBR207W \\
\hline & $\begin{array}{l}\text { Putative high affinity iron transporter; involved in transport of intravacuolar stores of iron; forms complex with } \\
\text { Fet5p; expression is regulated by iron; proposed to play indirect role in endocytosis; protein abundance increases in } \\
\text { response to DNA replication stress }\end{array}$ \\
\hline \multirow[t]{2}{*}{ SCS7 } & $115 \quad 3 \quad$ YMR272C fatty acid alpha-hydroxylase-FAH1 \\
\hline & $\begin{array}{l}\text { Sphingolipid alpha-hydroxylase; functions in the alpha-hydroxylation of sphingolipid-associated very long chain } \\
\text { fatty acids, has both cytochrome b5-like and hydroxylase/desaturase domains, not essential for growth }\end{array}$ \\
\hline \multirow[t]{2}{*}{ SAC1 } & 1162 YKL212W phosphatidylinositol-3-phosphatase SAC1-RSD1 \\
\hline & $\begin{array}{l}\text { Phosphatidylinositol phosphate (PtdInsP) phosphatase; involved in hydrolysis of PtdIns[4]P in the early and medial } \\
\text { Golgi; regulated by interaction with Vps } 74 \mathrm{p} \text {; ER localized transmembrane protein which cycles through the Golgi; } \\
\text { involved in protein trafficking and processing, secretion, and cell wall maintenance; regulates sphingolipid } \\
\text { biosynthesis through the modulation of PtdIns }(4) \mathrm{P} \text { metabolism }\end{array}$ \\
\hline \multirow[t]{2}{*}{ TNA1 } & $117 \quad 12 \quad$ YGR260W $\quad$ YGR260W \\
\hline & $\begin{array}{l}\text { High affinity nicotinic acid plasma membrane permease; responsible for uptake of low levels of nicotinic acid; } \\
\text { expression of the gene increases in the absence of extracellular nicotinic acid or para-aminobenzoate (PABA) }\end{array}$ \\
\hline \multirow[t]{2}{*}{ YGR149W } & $118 \quad 7 \quad$ YGR149W $\quad$ YGR149W \\
\hline & Putative protein of unknown function; predicted to be an integal membrane protein \\
\hline \multirow{2}{*}{ TPO1 } & YLL028W YLL028W \\
\hline & $\begin{array}{l}\text { Polyamine transporter of the major facilitator superfamily; member of the } 12 \text {-spanner drug: } \mathrm{H}(+) \text { antiporter } \mathrm{DHA} 1 \\
\text { family; recognizes spermine, putrescine, and spermidine; catalyzes uptake of polyamines at alkaline } \mathrm{pH} \text { and } \\
\text { excretion at acidic pH; during oxidative stress exports spermine, spermidine from the cell, which controls timing of } \\
\text { expression of stress-responsive genes; phosphorylation enhances activity and sorting to the plasma membrane }\end{array}$ \\
\hline \multirow[t]{2}{*}{ UBC6 } & YER100W E2 ubiquitin-conjugating protein UBC6-DOA2 \\
\hline & $\begin{array}{l}\text { Ubiquitin-conjugating enzyme involved in ERAD; located at the cytosolic side of the ER membrane; tail region } \\
\text { contains a transmembrane segment at the C-terminus; substrate of the ubiquitin-proteasome pathway; } \\
\text { ER-associated protein degradation is also known as ERAD }\end{array}$ \\
\hline \multirow[t]{2}{*}{ FTR1 } & YER145C high-affinity iron permease FTR1 \\
\hline & $\begin{array}{l}\text { High affinity iron permease; involved in the transport of iron across the plasma membrane; forms complex with } \\
\text { Fet3p; expression is regulated by iron; protein abundance increases in response to DNA replication stress }\end{array}$ \\
\hline \multirow[t]{2}{*}{ GAB1 } & $122 \quad 8 \quad$ YLR459W $\quad$ CDC91 \\
\hline & $\begin{array}{l}\text { GPI transamidase subunit; involved in attachment of glycosylphosphatidylinositol (GPI) anchors to proteins; may } \\
\text { have a role in recognition of the attachment signal or of the lipid portion of GPI }\end{array}$ \\
\hline \multirow[t]{2}{*}{ MEP2 } & ammonium permease MEP2 \\
\hline & $\begin{array}{l}\text { Ammonium permease involved in regulation of pseudohyphal growth; belongs to a ubiquitous family of cytoplasmic } \\
\text { membrane proteins that transport only ammonium }(\mathrm{NH} 4+) \text {; expression is under the nitrogen catabolite repression } \\
\text { regulation }\end{array}$ \\
\hline
\end{tabular}




\section{Ranked list, Top50 (TM-containing, ER-localized only), BLAST threshold: 1e-2}

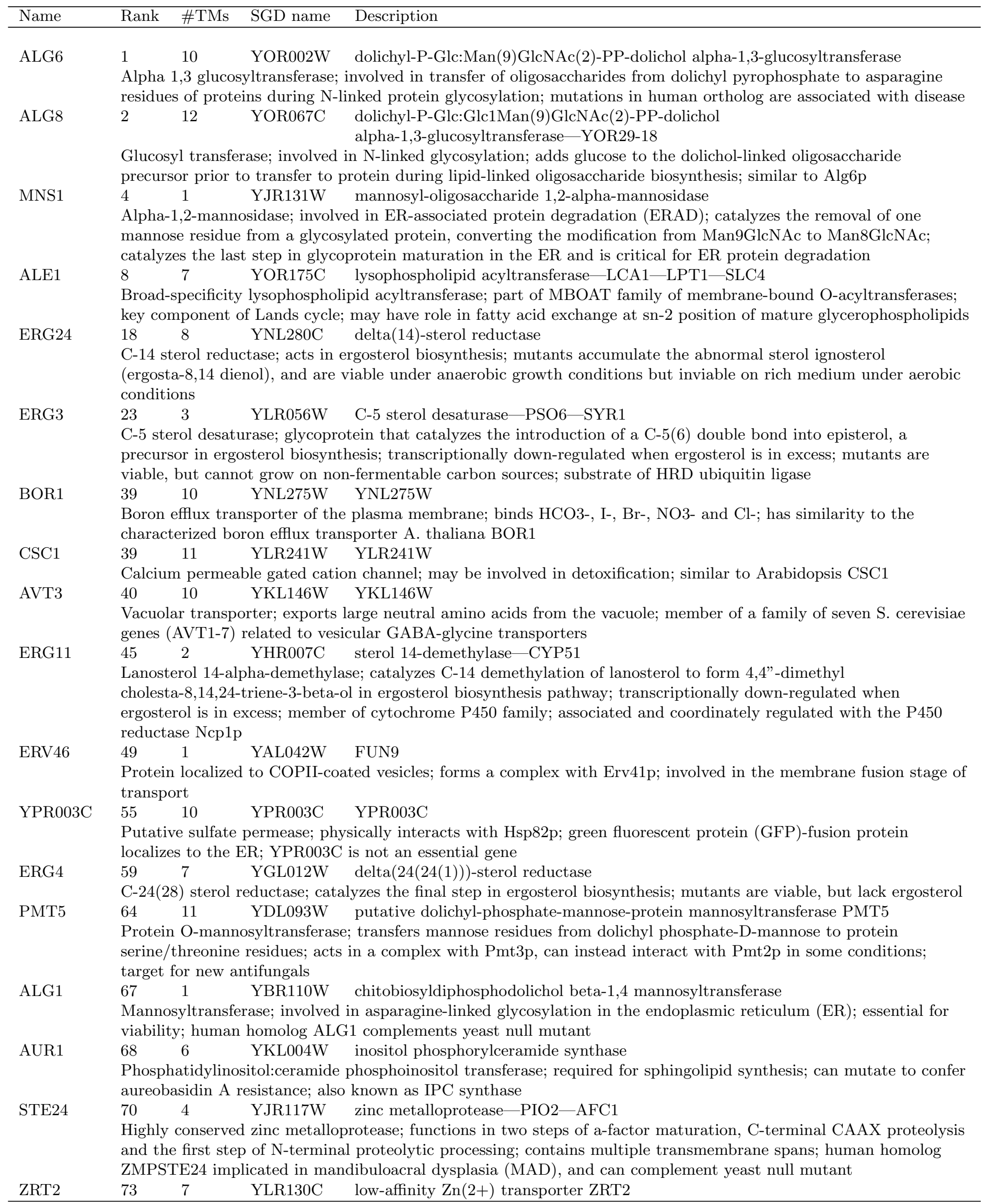




\begin{tabular}{llll}
\hline Name & Rank \#TMs & SGD name & Description \\
\hline
\end{tabular}

Low-affinity zinc transporter of the plasma membrane; transcription is induced under low-zinc conditions by the Zap1p transcription factor

MEP3 $\quad 74 \quad 11 \quad$ YPR138C ammonium permease MEP3

Ammonium permease of high capacity and low affinity; belongs to a ubiquitous family of cytoplasmic membrane proteins that transport only ammonium $(\mathrm{NH} 4+)$; expression is under the nitrogen catabolite repression regulation ammonia permease; MEP3 has a paralog, MEP1, that arose from the whole genome duplication

$\begin{array}{lllll}\text { ZRT1 } & 80 & 8 & \text { YGL255W high-affinity Zn(2+) transporter ZRT1 }\end{array}$

High-affinity zinc transporter of the plasma membrane; responsible for the majority of zinc uptake; transcription is induced under low-zinc conditions by the Zap1p transcription factor

$\begin{array}{lllll}\text { ATG15 } & 83 & 1 & \text { YCR068W triglyceride lipase ATG15-CVT17-AUT5 }\end{array}$

Putative lipase required for lysis of autophagic and Cvt bodies; targeted to intravacuolar vesicles during autophagy via the multivesicular body (MVB) pathway; required for the maintenance of lipid droplet quantity after the diauxic shift; regulates lipolysis

MEP1 $84 \quad 11 \quad$ YGR121C ammonium permease MEP1-AMT1 Ammonium permease; belongs to a ubiquitous family of cytoplasmic membrane proteins that transport only ammonium (NH4+); expression is under the nitrogen catabolite repression regulation; human homolog RHCG complements yeast null mutant; mutations in human homolog RHCG implicated in metabolic acidosis; MEP1 has a paralog, MEP3, that arose from the whole genome duplication

$\begin{array}{llll}\text { ERG9 } & 86 & 1 & \text { YHR190W bifunctional farnesyl-diphosphate farnesyltransferase/squalene synthase }\end{array}$ Farnesyl-diphosphate farnesyl transferase (squalene synthase); joins two farnesyl pyrophosphate moieties to form squalene in the sterol biosynthesis pathway

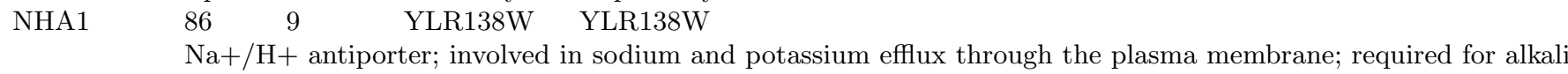
cation tolerance at acidic $\mathrm{pH}$

ALG3 $88 \quad 9 \quad$ YBL082C dolichyl-P-Man:Man(5)GlcNAc(2)-PP-dolichol

alpha-1,3-mannosyltransferase-RHK1

Dolichol-P-Man dependent alpha(1-3) mannosyltransferase; involved in the synthesis of dolichol-linked oligosaccharide donor for N-linked glycosylation of proteins

$\begin{array}{lllll}\text { LAC1 } & 93 & 7 & \text { YKL008C } & \text { sphingosine N-acyltransferase LAC1-DGT1 }\end{array}$

Ceramide synthase component; involved in synthesis of ceramide from C26(acyl)-coenzyme A and dihydrosphingosine or phytosphingosine, functionally equivalent to Lag1p; LAC1 has a paralog, LAG1, that arose from the whole genome duplication

$\begin{array}{lllll}\text { CBR1 } & 95 & 1 & \text { YIL043C } & \text { CBR5 }\end{array}$ Cytochrome b reductase; not essential for viability; also detected in mitochondria; mutation in conserved NADH binding domain of the human ortholog results in type I methemoglobinemia

DGA1 $\quad 100 \quad 1 \quad$ YOR245C diacylglycerol O-acyltransferase

Diacylglycerol acyltransferase; catalyzes the terminal step of triacylglycerol (TAG) formation, acylates diacylglycerol using acyl-CoA as an acyl donor; Lro1p and Dga1p can O-acylate ceramides; localized to lipid particles

VCX1 $101 \quad 11 \quad$ YDL128W MNR1-HUM1

Vacuolar membrane antiporter with $\mathrm{Ca} 2+/ \mathrm{H}+$ and $\mathrm{K}+/ \mathrm{H}+$ exchange activity; involved in control of cytosolic $\mathrm{Ca} 2+$ and $\mathrm{K}+$ concentrations; has similarity to sodium/calcium exchangers, including the bovine $\mathrm{Na}+/ \mathrm{Ca} 2+, \mathrm{K}+$ antiporter

$\begin{array}{lllll}\text { YKR051W } & 102 & 7 & \text { YKR051W } & \text { YKR051W }\end{array}$

Putative protein of unknown function

GWT1 $108 \quad 12 \quad$ YJL091C YJL091C

Protein involved in the inositol acylation of GlcN-PI; the inositol acylation of glucosaminyl phosphatidylinositol (GlcN-PI) forms glucosaminyl(acyl)phosphatidylinositol (GlcN(acyl)PI), an intermediate in the biosynthesis of glycosylphosphatidylinositol (GPI) anchors

PGA3 $\quad 108 \quad 2 \quad$ YML125C NQR1

Putative cytochrome b5 reductase, localized to the plasma membrane; may be involved in regulation of lifespan; required for maturation of Gas1p and Pho8p, proposed to be involved in protein trafficking; PGA3 has a paralog, AIM33, that arose from the whole genome duplication

ERG1 $110 \quad 2 \quad$ YGR175C squalene monooxygenase

Squalene epoxidase; catalyzes the epoxidation of squalene to 2,3-oxidosqualene; plays an essential role in the ergosterol-biosynthesis pathway and is the specific target of the antifungal drug terbinafine

$\begin{array}{lllll}\text { LAG1 } & 112 & 7 & \text { YHL003C } & \text { sphingosine N-acyltransferase LAG1 }\end{array}$ Ceramide synthase component; involved in synthesis of ceramide from C26(acyl)-coenzyme A and dihydrosphingosine or phytosphingosine, functionally equivalent to Lac1p; forms ER foci upon DNA replication stress; homolog of human CERS2, a tumor metastasis suppressor gene whose silencing enahnces invasion/metastasis of prostate cancer cells; LAG1 has a paralog, LAC1, that arose from the whole genome duplication 


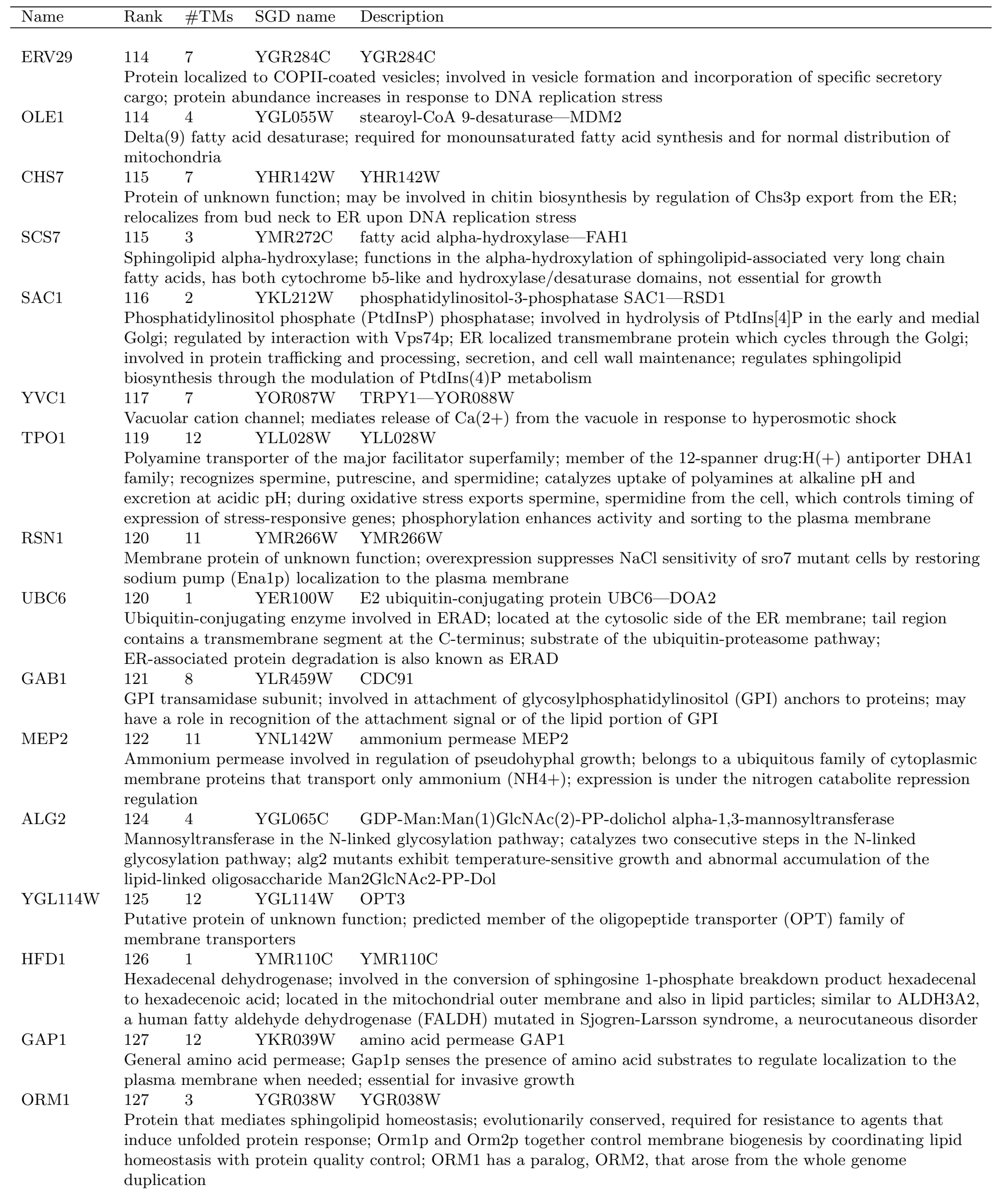




\section{Ranked list, Top50 (TM-containing, ER-localized only), BLAST threshold: 1e-10}

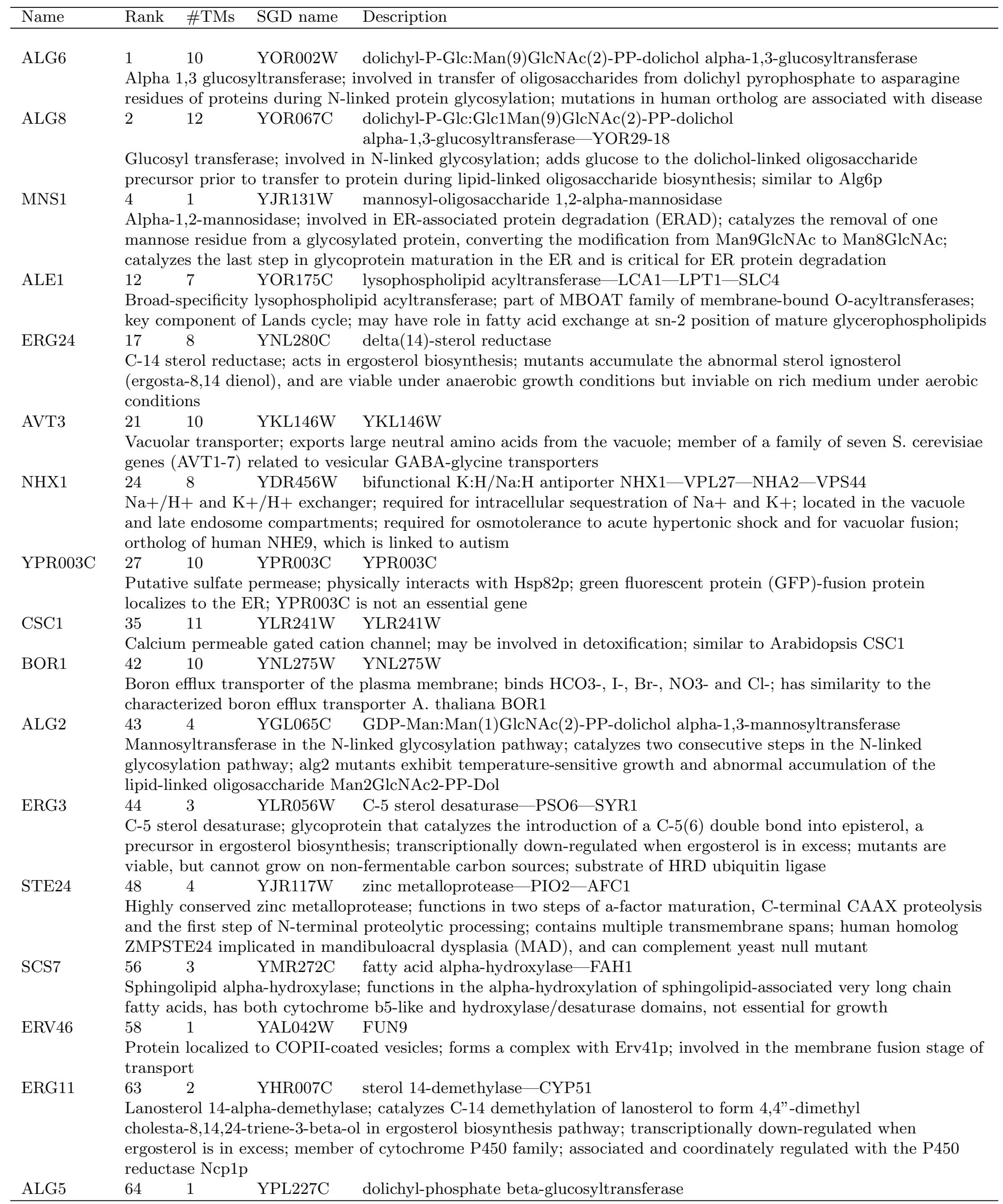




\begin{tabular}{|c|c|}
\hline Name & \#TMs $\quad$ SGD name \\
\hline & $\begin{array}{l}\text { UDP-glucose:dolichyl-phosphate glucosyltransferase; involved in asparagine-linked glycosylation in the endoplasmic } \\
\text { reticulum }\end{array}$ \\
\hline \multirow[t]{2}{*}{ ALG1 } & chitobiosyldiphosphodolichol beta-1,4 mannosyltransferase \\
\hline & $\begin{array}{l}\text { Mannosyltransferase; involved in asparagine-linked glycosylation in the endoplasmic reticulum (ER); essential for } \\
\text { viability; human homolog ALG1 complements yeast null mutant }\end{array}$ \\
\hline \multirow[t]{2}{*}{ GWT1 } & $70 \quad 12 \quad$ YJL091C $\quad$ YJL091C \\
\hline & $\begin{array}{l}\text { Protein involved in the inositol acylation of GlcN-PI; the inositol acylation of glucosaminyl phosphatidylinositol } \\
\text { (GlcN-PI) forms glucosaminyl(acyl)phosphatidylinositol (GlcN(acyl)PI), an intermediate in the biosynthesis of } \\
\text { glycosylphosphatidylinositol (GPI) anchors }\end{array}$ \\
\hline \multirow[t]{2}{*}{ LAC1 } & $72 \quad 7 \quad$ YKL008C $\quad$ sphingosine N-acyltransferase LAC1-DGT1 \\
\hline & $\begin{array}{l}\text { Ceramide synthase component; involved in synthesis of ceramide from C26(acyl)-coenzyme A and } \\
\text { dihydrosphingosine or phytosphingosine, functionally equivalent to Lag1p; LAC1 has a paralog, LAG1, that arose } \\
\text { from the whole genome duplication }\end{array}$ \\
\hline \multirow[t]{2}{*}{ ZRT2 } & $\begin{array}{llll}73 & 7 & \text { YLR130C } & \text { low-affinity } \mathrm{Zn}(2+) \text { transporter ZRT2 }\end{array}$ \\
\hline & $\begin{array}{l}\text { Low-affinity zinc transporter of the plasma membrane; transcription is induced under low-zinc conditions by the } \\
\text { Zap1p transcription factor }\end{array}$ \\
\hline \multirow[t]{2}{*}{ PMT5 } & $77 \quad 11 \quad$ YDL093W putative dolichyl-phosphate-mannose-protein mannosyltransferase PMT5 \\
\hline & $\begin{array}{l}\text { Protein O-mannosyltransferase; transfers mannose residues from dolichyl phosphate-D-mannose to protein } \\
\text { serine/threonine residues; acts in a complex with Pmt3p, can instead interact with Pmt2p in some conditions; } \\
\text { target for new antifungals }\end{array}$ \\
\hline \multirow[t]{2}{*}{ VCX1 } & $79 \quad 11 \quad$ YDL128W $\quad$ MNR1-HUM1 \\
\hline & $\begin{array}{l}\text { Vacuolar membrane antiporter with } \mathrm{Ca} 2+/ \mathrm{H}+\text { and } \mathrm{K}+/ \mathrm{H}+\text { exchange activity; involved in control of cytosolic } \\
\mathrm{Ca} 2+\text { and } \mathrm{K}+\text { concentrations; has similarity to sodium/calcium exchangers, including the bovine } \mathrm{Na}+/ \mathrm{Ca} 2+, \mathrm{K}+ \\
\text { antiporter }\end{array}$ \\
\hline \multirow[t]{2}{*}{ AUR1 } & $82 \quad 6 \quad$ YKL004W inositol phosphorylceramide synthase \\
\hline & $\begin{array}{l}\text { Phosphatidylinositol:ceramide phosphoinositol transferase; required for sphingolipid synthesis; can mutate to confer } \\
\text { aureobasidin A resistance; also known as IPC synthase }\end{array}$ \\
\hline \multirow[t]{2}{*}{ CBR1 } & $82 \quad 1 \quad$ YIL043C $\quad$ CBR5 \\
\hline & $\begin{array}{l}\text { Cytochrome b reductase; not essential for viability; also detected in mitochondria; mutation in conserved NADH } \\
\text { binding domain of the human ortholog results in type I methemoglobinemia }\end{array}$ \\
\hline \multirow[t]{2}{*}{ SAC1 } & 832 YKL212W phosphatidylinositol-3-phosphatase SAC1-RSD1 \\
\hline & $\begin{array}{l}\text { Phosphatidylinositol phosphate (PtdInsP) phosphatase; involved in hydrolysis of PtdIns[4]P in the early and medial } \\
\text { Golgi; regulated by interaction with Vps74p; ER localized transmembrane protein which cycles through the Golgi; } \\
\text { involved in protein trafficking and processing, secretion, and cell wall maintenance; regulates sphingolipid } \\
\text { biosynthesis through the modulation of PtdIns }(4) \mathrm{P} \text { metabolism }\end{array}$ \\
\hline \multirow[t]{2}{*}{ ZRT1 } & $83 \quad 8 \quad$ YGL255W high-affinity Zn $(2+)$ transporter ZRT1 \\
\hline & $\begin{array}{l}\text { High-affinity zinc transporter of the plasma membrane; responsible for the majority of zinc uptake; transcription is } \\
\text { induced under low-zinc conditions by the Zap1p transcription factor }\end{array}$ \\
\hline \multirow[t]{2}{*}{ ATG15 } & $85 \quad 1 \quad$ YCR068W triglyceride lipase ATG15-CVT17-AUT5 \\
\hline & $\begin{array}{l}\text { Putative lipase required for lysis of autophagic and Cvt bodies; targeted to intravacuolar vesicles during autophagy } \\
\text { via the multivesicular body (MVB) pathway; required for the maintenance of lipid droplet quantity after the } \\
\text { diauxic shift; regulates lipolysis }\end{array}$ \\
\hline \multirow[t]{2}{*}{ ERG4 } & $92 \quad 7 \quad$ YGL012W delta $(24(24(1)))$-sterol reductase \\
\hline & C-24(28) sterol reductase; catalyzes the final step in ergosterol biosynthesis; mutants are viable, but lack ergosterol \\
\hline \multirow[t]{2}{*}{ NHA1 } & $92 \quad 9 \quad$ YLR138W YLR138W \\
\hline & $\begin{array}{l}\mathrm{Na}+/ \mathrm{H}+\text { antiporter; involved in sodium and potassium efflux through the plasma membrane; required for alkali } \\
\text { cation tolerance at acidic } \mathrm{pH}\end{array}$ \\
\hline \multirow[t]{2}{*}{ LAG1 } & $93 \quad 7 \quad$ YHL003C $\quad$ sphingosine N-acyltransferase LAG1 \\
\hline & $\begin{array}{l}\text { Ceramide synthase component; involved in synthesis of ceramide from C26(acyl)-coenzyme A and } \\
\text { dihydrosphingosine or phytosphingosine, functionally equivalent to Lac1p; forms ER foci upon DNA replication } \\
\text { stress; homolog of human CERS2, a tumor metastasis suppressor gene whose silencing enahnces invasion/metastasis } \\
\text { of prostate cancer cells; LAG1 has a paralog, LAC1, that arose from the whole genome duplication }\end{array}$ \\
\hline \multirow[t]{2}{*}{ MEP3 } & YPR138C ammonium permease MEP3 \\
\hline & $\begin{array}{l}\text { Ammonium permease of high capacity and low affinity; belongs to a ubiquitous family of cytoplasmic membrane } \\
\text { proteins that transport only ammonium (NH4+); expression is under the nitrogen catabolite repression regulation } \\
\text { ammonia permease; MEP3 has a paralog, MEP1, that arose from the whole genome duplication }\end{array}$ \\
\hline \multirow[t]{2}{*}{ LRO1 } & $95 \quad 1 \quad$ YNR008W phospholipid:diacylglycerol acyltransferase \\
\hline & $\begin{array}{l}\text { Acyltransferase that catalyzes diacylglycerol esterification; one of several acyltransferases that contribute to } \\
\text { triglyceride synthesis; Lro1p and Dga1p can O-acylate ceramides; putative homolog of human lecithin cholesterol } \\
\text { acyltransferase }\end{array}$ \\
\hline
\end{tabular}




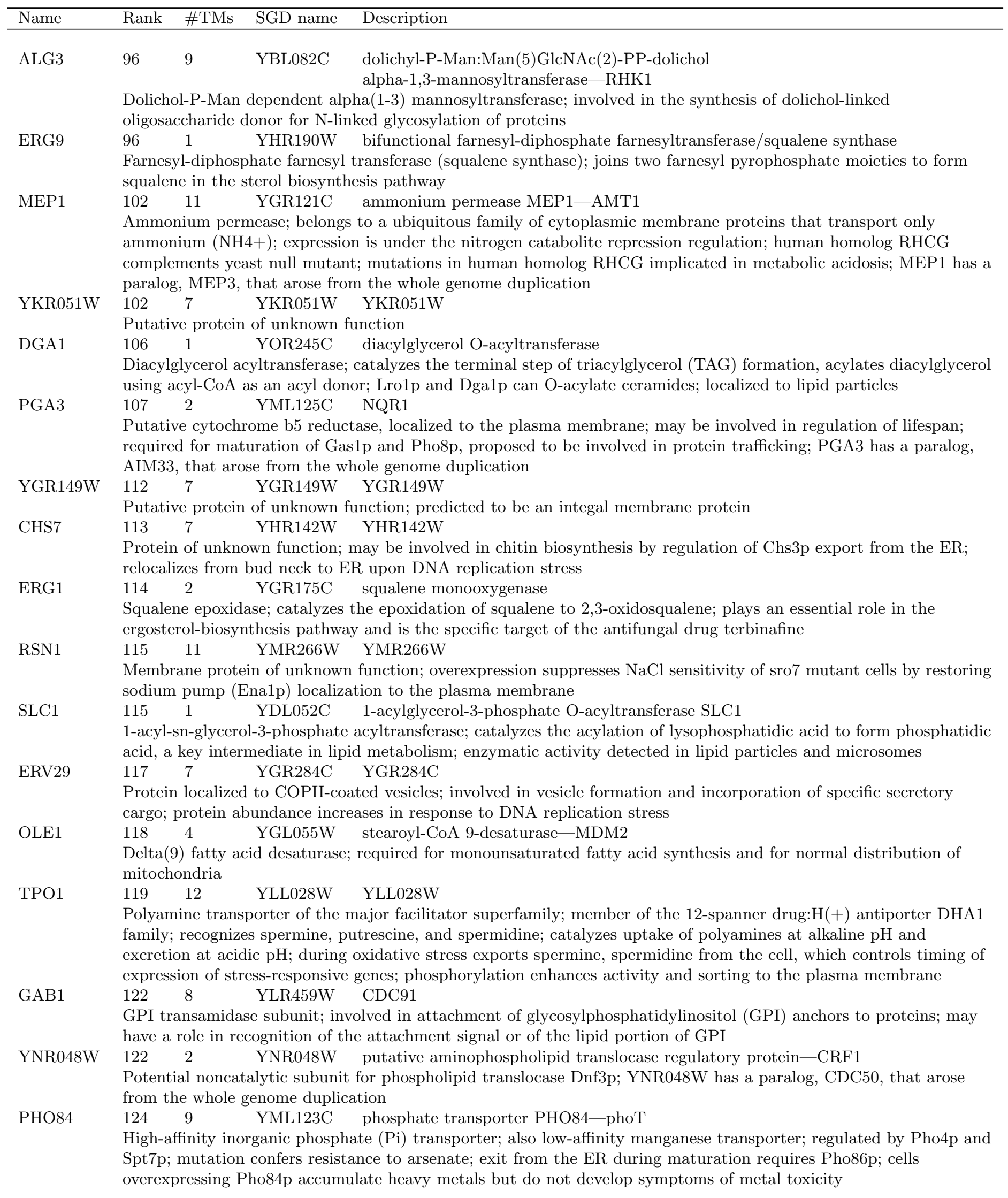

